\section{Neues rund ums Blasenkarzinom}

Z Diagnostik und Therapie des Blasenkarzinoms, dem mit rund 27.000 Neuerkrankungen pro Jahr zweithäufigsten urologischen Tumor, gab es in den letzten Jahren einige Neuerungen. Diese seien zum Teil noch experimentell, wie PD Dr. Georg Bartsch, Frankfurt, berichtete. So zeichnen sich mit der RamanSpektroskopie und dem Narrow-Band-Imaging neue Methoden $\mathrm{ab}$, die in Zukunft die Diagnostik erleichtern könnten. Hinsichtlich des oberflächlichen Harnblasenkarzinoms steht nach den beiden großen EORTC-Studien fest, dass BCG (Bacillus Calmette-Guérin) der Standard bleibt. Dr. Hans-Martin Fritsche, Regensburg, stellte eine erste prospektive Untersuchung der transurethralen enbloc-Dissektion von Urothelkarzinomen der Harnblase mittels Hochdruck-Wasserstrahl vor. Mit dem 30-bar-Wasserstrahl konnten sämtliche Tumoren en-bloc von der Blasenwand abgelöst werden. Rezidiv- und Progressrate wurden in dieser Studie jedoch noch nicht untersucht.

Zudem bestehen beim invasiven Karzinom zur operativen Therapie noch offene Fragen wie zum Ausmaß der Lymphadenektomie im Rahmen der Zystektomie. Diese sollen im Rahmen der LEA-Studie der Arbeitsgemeinschaft Urologische Onkologie (AUO) beantwortet werden. Die Rekrutierung für die weltweit erste große Studie zu diesem Thema wurde im August 2010 mit der Aufnahme von 485 Patienten abgeschlossen. Eine erste Zwischenauswertung liegt für 375 Patienten vor, allerdings sind dort die endgültigen Studienziele wie das progressionsfreie Überleben noch nicht vollständig ausgewertet. Bartsch erwartet, dass die Studie im nächsten Jahr vorgestellt werden kann. Bettina Reich

P 19: „Urothelkarzinom - Therapie fortgeschrittener Tumoren”

\section{CAPRA-Score ubiquitär einsetzbar}

$\mathrm{B}$ ei jedem sechsten Mann über 50 wird heute ein Prostatakarzinom diagnostiziert, aber in derselben Altersgruppe stirbt nur jeder 33. daran. Schwierig ist in jedem Fall die Therapieentscheidung, betonte Dr. Lars Budäus, Hamburg. Während es bei frühen Stadien möglicherweise zu einer Übertherapie durch lokale Behandlungen kommt, ist bei fortgeschrittenen Stadien - gerade bei aggressiven Tumoren - häufig eine Untertherapie feststellbar. Wichtig ist daher die Prädiktion des Rezidivrisikos. Dafür gibt es seit einigen Jahren den CAPRA-Score („cancer of the prostate risk assessment“). Damit werden dem Alter, PSA-Wert, Gleason-Score, der lokalen Tumorausdehnung und dem prozentualen Tumoranteil in der Biopsie Punkte zugeordnet. Bei null bis zwei Punkten ist aktive Überwachung möglich, bei drei bis vier eine lokale Therapie mit kurativer Zielsetzung und bei fünf bis sechs wird diese kurative Maßnahme durch eine adjuvante Hormontherapie ergänzt. Einen systemischen Androgenentzug erfordern sieben bis zehn Punkte. Allerdings wurde dieser Score auf Basis amerikanischer Patientendaten erstellt. Um den Score und seine Wertigkeit hierzulande zu überprüfen, wurden 3.209 europäische Patienten mit Prostatakarzinom untersucht. Zielsetzung der Studie war es, auch in Europa mithilfe des CAPRA-Scores präzise Vorhersagen der Metastasen-Entwicklung bei Prostatakrebspatienten zu machen. In dieser europäischen Kohorte lag die Vorhersagegenauigkeit bei 80,2\%. Daher folgerte Budäus: „Der CAPRA-Score ist ubiquitär einsetzbar und sollte auch hier zur Vorhersage von Metastasen genutzt werden."

Bettina Reich

\title{
Neue Strategien zur Nachwuchsförderung
}

$\mathrm{D}$ ie Zukunftsfähigkeit der Urologie kann nur sichergestellt werden, wenn qualifizierter Nachwuchs zur Verfügung steht. Junge Talente zu rekrutieren und $\mathrm{zu}$ fördern, hat sich die Deutsche Gesellschaft für Urologie (DGU) daher einmal mehr auf die Fahnen geschrieben.

Der Mangel an qualifiziertem Nachwuchs ist in der Urologie wie in den meisten anderen medizinischen Disziplinen ein drängendes Problem. Prof. Joachim Steffens, Präsident der DGU, hat daher die Nachwuchsförderung zu einem zentralen Thema des diesjährigen DGU-Kongresses gemacht. Die DGU hatte bereits 2009 mit der Vergabe von Forschungsstipendien (EisenbergerStipendium) eine umfassende Initiative gestartet, die nun durch die neu gegründete Juniorakademie ergänzt wird. Stu- denten im praktischen Jahr und junge Assistenzärzte sind die Zielgruppe dieser Akademie, die sich an den Erfahrungen aus der Wirtschaft und dem Management orientiert. In der Juniorakademie entwickeln erfahrene Kollegen aus Forschung, Klinik und Praxis Zukunftsperspektiven und skizzieren Berufsaussichten für die jungen Kollegen. Ferner werden Themen wie Vertrauenskultur, Umgang mit Fehlern und Risikomanagement behandelt.

Steffens appellierte an die ärztlichen Kollegen, aktiv zu werden. Talentmanagement könne nur gelingen, wenn sie die jungen Mitarbeiter durch eine Vorbildfunktion begeisterten. Talentmanagement sei ein Top-Thema für das Top-Management und könne nicht delegiert werden. Doch Führung zu übernehmen, werde bisher im Studium nicht vermittelt, erklärte Steffens. Nach seinen Worten müsse sich das Verhalten der urologischen Lehrer künftig noch mehr darauf konzentrieren, den ärztlichen Nachwuchs nachhaltig für das Fach zu motivieren. Wie das geht? Durch Wahrnehmung und Wertschätzung der jungen Kollegen, durch Einbeziehung in die verschiedenen Spezialitäten des Fachgebiets in Klinik, Forschungslabor und Praxis sowie durch Unterstützung bei der Karriereplanung. Steffens Fazit: „Nur wenn die Leidenschaft für das eigene Fachgebiet spürbar und die Begeisterung für die tägliche $\mathrm{He}$ rausforderung vermittelt wird, werden wir angehende, junge Kollegen für die Urologie gewinnen können.“ Dr. Claudia Mäck

Eröffnungs-Pressekonferenz 